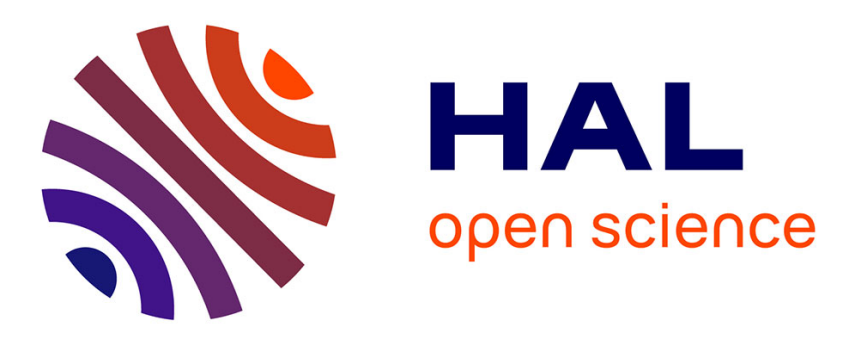

\title{
Analysis of drilling vibrations: A time-delay system approach
}

Islam Boussaada, Hugues Mounier, Silviu-Iulian Niculescu, Arben Cela

\section{To cite this version:}

Islam Boussaada, Hugues Mounier, Silviu-Iulian Niculescu, Arben Cela. Analysis of drilling vibrations: A time-delay system approach. 20th Mediteranean Conference on Control and Automation, Jul 2012, Barcelona, Spain. p. 610-614. hal-00832013

\section{HAL Id: hal-00832013 https://hal.science/hal-00832013}

Submitted on 9 Jun 2013

HAL is a multi-disciplinary open access archive for the deposit and dissemination of scientific research documents, whether they are published or not. The documents may come from teaching and research institutions in France or abroad, or from public or private research centers.
L'archive ouverte pluridisciplinaire $\mathbf{H A L}$, est destinée au dépôt et à la diffusion de documents scientifiques de niveau recherche, publiés ou non, émanant des établissements d'enseignement et de recherche français ou étrangers, des laboratoires publics ou privés. 


\title{
Analysis of Drilling vibrations: A Time-Delay System Approach
}

\author{
Islam Boussaada and Hugues Mounier and Silviu-Iulian Niculescu and Arben Cela
}

\begin{abstract}
The main purpose of this study is the description of the qualitative dynamical response of a rotary drilling system with a drag bit, using a model that takes into consideration the axial and the torsional vibration modes of the bit. The studied model, based on the interface bit-rock, contains a couple of wave equations with boundary conditions consisting of the angular speed and the axial speed at the top additionally to the angular and axial acceleration at the bit whose contain a realistic frictional torque. Our analysis is based on the center manifold Theorem and Normal forms theory whose allow us to simplify the model.
\end{abstract}

Index Terms - Drilling system, Vibrations analysis, Timedelay systems, Neutral systems, Functional differential equations, Center Manifold, Normal forms.

\section{INTRODUCTION}

Interconnected oscillatory systems often display what is called propagation phenomena, [13]. In general by Lossless propagation it is understood the phenomenon associated with long transmission lines for physical signals. In engineering, this problem is strongly related to electric and electronic applications, e.g. circuit structures consisting of multipoles connected through LC transmission lines, this can also be seen in steam or gaz flows or pressures and water pipes [21], [9], [23]. The mathematical model is described in all these cases by a mixed initial and boundary value problem for hyperbolic partial differential equations modeling the lossless propagation. The boundary conditions are of special type being in feedback connection with some system described by ordinary differential equation. This leads to the socalled derivative boundary conditions considered by Cooke \& Krumme [6] but also to the even more general boundary conditions of Abolina \& Myshkis described by Volterra operators, see [23]. Integration along characteristics of the hyperbolic partial differential equation is in fact the method of d'Alembert, it allows the association of certain system of functional equations to the mixed problem.

This paper is concerned by an application which can be modeled by such equations therefore the above idea is adopted, see [2], [8], [25], [26]. The analysis and modeling

I. Boussaada is with Laboratoire des signaux et systèmes, (L2S, UMR CNRS 8506), Supélec, 3 rue Joliot-Curie, 91192 Gif-sur-Yvette cedex, France and Laboratoire de Modélisation et Calcul Scientifique, Institut Polytechnique des Sciences Avancées, 7 rue Maurice Grandcoing, 94200 Ivry-sur-Seine, France is lam.boussaada@gmail . com

H. Mounier is with Laboratoire des signaux et systèmes, (L2S, UMR CNRS 8506), Supélec, 3 rue Joliot-Curie, 91192 Gif-sur-Yvette cedex, France Hugues. Mounierdlss. supelec. fr

S-I. Niculescu is with Laboratoire des signaux et systèmes, (L2S, UMR CNRS 8506), Supélec, 3 rue Joliot-Curie, 91192 Gif-sur-Yvette cedex, France Silviu.Niculescullss.supelec.fr

A. Cela is with Department of Embedded Systems, UPE, 93162 NoisyLe-Grand, France celaadesiee.fr of a rotary drilling vibrations is a topic whose economical interest has been renewed by recent oilfields discoveries leading to a growing literature, see for instance [24], [10], [20], [17], [19] and [18].

Throughout this paper, we would like first to contribute by improving the modelling of the drilling system by taking into account both axial and torsional vibrations and secondly by extending the qualitative analysis to the investigation of the nonlinear terms in the model. Indeed, the center manifold theorem and normal forms theory are applied to obtain a finite dimensional approximation that conserves the main dynamics of the physical system. Let us consider the following model for the axial vibrations $U$ and torsional vibrations $\Phi$ :

$$
\left\{\begin{array}{rl}
\partial_{t}^{2} U(t, s) & =c^{2} \partial_{s}^{2} U(t, s) \\
E \Gamma \partial_{s} U(t, 0) & =\alpha \partial_{t} U(t, 0)-H(t) \\
M \partial_{t}^{2} U(t, L) & =-E \Gamma \partial_{s} U(t, L)+F\left(\partial_{t} U(t, L)\right)
\end{array},\right.
$$

and

$$
\left\{\begin{array}{rl}
\partial_{t}^{2} \Phi(t, s) & =\tilde{c}^{2} \partial_{s}^{2} \Phi(t, s) \\
G \Sigma \partial_{s} \Phi(t, 0) & =\beta \partial_{t} \Phi(t, 0)-\Omega(t) \\
J \partial_{t}^{2} \Phi(t, L) & =-G \Sigma \partial_{s} \Phi(t, L)+\tilde{F}\left(\partial_{t} U(t, L)\right)
\end{array},\right.
$$

where in equation (1) $H$ is the brake motor control and $\alpha \partial_{t} U(t, 0)$ represents a friction force of viscous type. For equation (2), the right hand side of the second equation designate the difference between the motor speed and rotational speed of the first pipe. The physical parameters of the model (1)-(2) are: $G$ is the shear modulus the for the drillstring steel and $E$ is the elasticity Young's modulus, then the wave speeds can be expressed by $c=\sqrt{E / \rho}$ and $\tilde{c}=\sqrt{G / \rho}$ and $J$ is the inertia $J=M r^{2}$ where $r$ is taken as the averaged radius of drillpipe and $\Gamma$ is the averaged section of the drillpipe and $\Sigma$ is the quadratic momentum. Those parameters are taken following the the numerical settings presented in the Appendix. The nonlinear aspect of the model is considered by taking functions $F$ and $\tilde{F}$ in the form: $z \mapsto p k z /\left(k^{2} z^{2}+\zeta\right)$ where the parameters $p, k, \zeta$ are some positive integer responsible of the sharpness of the top angle of the friction force graph and $p$ is some parameter deciding the amplitude of the friction force such that $0<\zeta \ll 1$ and $0<k<1$. Moreover, the behavior of the chosen friction model is close from the empirical model: the white friction force but is more handleable; which can be very useful in experimental identifications. Note also that the proposed model can be expanded to Taylor sum, this fact is very important when the aim is to give accurate approximation at any fixed order. The chosen 
functions have a close behavior to the one used in [3] for modelling the friction. The second originality of the present contribution is an analytical study of the drilling model as functional differential equations of neutral type and based on the qualitative theory; Center Manifold Theorem [5] and Normal Forms Theory [11]. Indeed, most of the references concerned by partial differential equations (PDE) or Delay differential equations (DDE) models for the drilling problem have a numerical analysis character. In this work we reduce the considered PDE model to a singularly perturbed system of ordinary differential equations (ODE) in like BogdanovTakens configuration (double zero eigenvalues). Furthermore, to the best of the authors knowledge, this type of singularity never studied for NDDE depending on parameter, thus we extend the methodology for computing the center manifold which allows us to establish the linear stability and the bifurcation elements. Similar result can be found in [12] where the analysis of a physiological control model of DDE with double-zero eigenvalue singularity. This study have the same spirit of the results of Bogdanov and Takens for ODEs. We refer the reader to [14], [11] for elements of Bifurcation theory.

The remaining part of the paper is organized as follows. The second section is concerned by preliminaries, we describe the standard procedure for reducing the PDE drillstring model to a Neutral Delay differential equations (NDDE). In the third section, entitled Dynamics Analysis, we establish linear stability analysis and apply bifurcation results for Bogdanov-Takens singularity. The methodological scheme described in [1], [4] is extended to the study of the parametrized model of neutral type. For the sake of selfcontainment, we report in the Appendix, a table for the numerical settings for the parameters used in (1)-(2), then we present elements of the qualitative theory of differential equations, entitled Model reduction, which comprises the outlines of a methodology enabling to approximate a system of NDDE by a system of ordinary differential equations (Center Variety) and then to study local bifurcations (Normal Forms).

\section{PRELIMINARIES}

For the sake of self-containment, in the sequel we describe a standard procedure allowing to transform the considered PDE model to a delay system of Neutral type. To the best of the authors knowledge, this was presented for the first time in [6], see also [2] and [16].

\section{A. Axial vibrations}

First, let us consider the subsystem (1).

The change of variables $\xi=t+c s$ and $\eta=t-c s$ gives from the top equation of (1) $\partial^{2}{ }_{\xi \eta} U(t, s)=0$ which leads to separate the variables, i.e. $U(\xi, \eta)=\varphi(\xi)+\psi(\eta)$.

Then

$$
\partial_{t} U=\frac{\partial}{\partial \xi}(\varphi)+\frac{\partial}{\partial \eta}(\psi), \text { and } \partial_{s} U=c \frac{\partial}{\partial \xi}(\varphi)-c \frac{\partial}{\partial \eta}(\psi)
$$

Substituting this into the two last equations (boundary conditions) of (1) and introducing $\tau$ such that $\tau=c L$ we obtain

$$
\frac{E \Gamma}{\alpha} \partial_{s} U(t, 0)=\partial_{t} U(t, 0)-\frac{1}{\alpha} H(t) \text {. }
$$

To simplify notations, from now we adopt $(\cdot)$ for $\left(\partial_{t}=\frac{d}{d t}\right)$.

$H(t)=(\alpha-c E \Gamma) \dot{\varphi}(t)+(\alpha+c E \Gamma) \dot{\psi}(t)=A \dot{\varphi}(t)+B \dot{\psi}(t)$

such that $A=\alpha-c E \Gamma$ and $B=\alpha+c E \Gamma$.

$$
\begin{aligned}
M \ddot{U}(t, L) & =M \ddot{\varphi}(t+\tau)+M \ddot{\psi}(t-\tau) \\
& =-E \Gamma c \dot{\varphi}(t+\tau)+E \Gamma c \dot{\psi}(t-\tau) \\
& +F(\dot{\varphi}(t+\tau)+\dot{\psi}(t-\tau)) .
\end{aligned}
$$

Let $v$ be the axial vibration at the bit

$$
v(t)=U(t, L)=\varphi(t+\tau)+\psi(t-\tau),
$$

which gives

$$
\varphi(t)=v(t-\tau)-\psi(t-2 \tau) .
$$

Equality (4) with (7) give

$$
H(t-\tau)=A(\dot{v}(t-2 \tau)-\dot{\psi}(t-3 \tau))+B \dot{\psi}(t-\tau) .
$$

Equality (5) with (7) give

$$
M \ddot{v}(t)=-E \Gamma c \dot{v}(t)+2 E \Gamma c \dot{\psi}(t-\tau)+F(\dot{v}(t)) .
$$

This last equality gives

$$
\dot{\psi}(t-\tau)=\frac{M \ddot{v}(t)+E \Gamma c \dot{v}(t)-F(\dot{v}(t))}{2 E \Gamma c} .
$$

One obtains the first neutral delay differential equation by substituting (10) into (8):

$$
\begin{aligned}
\ddot{v}(t)-\frac{A}{B} \ddot{v}(t-2 \tau) & =-\frac{E \Gamma c}{M} \dot{v}(t)-\frac{A E \Gamma c}{M B} \dot{v}(t-2 \tau) \\
& +\frac{1}{M} F(\dot{v}(t))-\frac{A}{B M} F(\dot{v}(t-2 \tau)) \\
& +\frac{2 E \Gamma c}{B M} H(t-\tau)
\end{aligned}
$$

\section{B. Torsional vibrations}

Let us deal with the torsional vibrations and consider the subsystem (2). We use the change of variables $\alpha=t+\tilde{c} s$ and $\beta=t-\tilde{c} s$ and we separate the variables $\Phi(\alpha, \beta)=$ $\tilde{\varphi}(\alpha)+\tilde{\psi}(\beta)$. Then

$$
\partial_{t} \Phi=\frac{\partial}{\partial \alpha}(\tilde{\varphi})+\frac{\partial}{\partial \beta}(\tilde{\psi})
$$

and

$$
\partial_{s} \Phi=\tilde{c} \frac{\partial}{\partial \alpha}(\tilde{\varphi})-\tilde{c} \frac{\partial}{\partial \beta}(\tilde{\psi}) .
$$

Now let $w$ be the torsional vibration at the bit, by the same way as for the axial vibrations, we obtain

$$
\begin{aligned}
\ddot{w}(t)-\frac{C}{D} \ddot{w}(t-2 \tilde{\tau}) & =-\frac{\tilde{c} G \Sigma}{J} \dot{w}(t)-\frac{\tilde{c} C G \Sigma}{D J} \dot{w}(t-2 \tilde{\tau}) \\
& +\frac{1}{J} \tilde{F}(\dot{v}(t))-\frac{C}{D J} \tilde{F}(\dot{v}(t-2 \tilde{\tau})) \\
& +\frac{2 \tilde{c} G \Sigma}{D J} \Omega(t-\tilde{\tau})
\end{aligned}
$$


such that $C=\beta-\tilde{c} G \Sigma$ and $D=\beta+\tilde{c} G \Sigma$. Now let us consider the two obtained neutral equations (11)-(12). To obtain the NDDE dimensionless form of (1)-(2) one may adopt the following units of length, time and torque the quantities $L, T=L / c$ and $E \Gamma / L$, thus system (11)-(12) is written

$$
\left\{\begin{array}{l}
\ddot{v}(t)-\frac{\alpha-1}{\alpha+1} \ddot{v}(t-2)= \\
-\frac{1}{M} \dot{v}(t)-\frac{\alpha-1}{M(\alpha+1)} \dot{v}(t-2)+\frac{2}{M(\alpha+1)} H(t-1) \\
+\frac{1}{M} F(\dot{v}(t))-\frac{\alpha-1}{M(\alpha+1)} F(\dot{v}(t-2)) \\
\ddot{w}(t)-\frac{c E \Gamma \beta-\tilde{c} G \Sigma}{c E \Gamma \beta+\tilde{c} G \Sigma} \ddot{w}(t-2 \tilde{\tau})= \\
-\frac{\tilde{c} G \Sigma}{c E \Gamma J} \dot{w}(t)-\frac{\tilde{c} G \Sigma}{c E \Gamma J} \frac{c E \Gamma-\tilde{c} G \Sigma}{c E \Gamma \beta+\tilde{c} G \Sigma} \dot{w}(t-2 \tilde{\tau}) \\
+\frac{1}{J} \tilde{F}(\dot{v}(t))-\frac{c E \Gamma \beta-\tilde{c} G \Sigma}{J(c E \Gamma \beta+\tilde{c} G \Sigma)} \tilde{F}(\dot{v}(t-2 \tilde{\tau})) \\
+\frac{2 \tilde{c} G \Sigma}{J(c E \Gamma \beta+\tilde{c} G \Sigma)} \Omega(t-\tilde{\tau})
\end{array}\right.
$$

where $\tilde{\tau}$ is the ratio of the speeds $\tilde{\tau}=\frac{\tilde{c}}{c}$.

\section{DyNAMICS ANALYSIS OF THE UNCONTROLLED DRILLING SYSTEM}

Let us consider the normalized and uncontrolled system, i.e. $\Omega=H=0$.

\section{A. Linearized Stability and Bifurcation Analysis}

Let denote by $x_{1}$ the axial vibrations speed $x_{1}=\dot{v}$ and by $x_{2}$ the angular vibrations speed $x_{2}=\dot{w}$ and adopt the matrix representation of the linear part of the above system where $x=\left(x_{1}, x_{2}\right)^{T}$

$$
\left\{\begin{aligned}
\dot{x}(t)= & D_{1} \dot{x}(t-2)+D_{2} \dot{x}\left(t-\frac{2 \tilde{c}}{c}\right)+A_{0} x(t)+A_{1} x(t-2) \\
& +A_{2} x\left(t-\frac{2 \tilde{c}}{c}\right)+\mathcal{F}\left(x(t), x(t-2), x\left(t-\frac{2 \tilde{c}}{c}\right)\right)
\end{aligned}\right.
$$

where $\mathcal{F}$ is the nonlinear part of the system (13)

$$
\begin{gathered}
D_{1}=\left[\begin{array}{cc}
d_{1,1,1} & 0 \\
0 & 0
\end{array}\right], D_{2}=\left[\begin{array}{cc}
0 & 0 \\
0 & d_{2,2,2}
\end{array}\right], \\
A_{0}=\left[\begin{array}{cc}
a_{0,1,1} & 0 \\
a_{0,2,1} & a_{0,2,2}
\end{array}\right], A_{1}=\left[\begin{array}{cc}
a_{1,1,1} & 0 \\
0 & 0
\end{array}\right], \\
A_{2}=\left[\begin{array}{cc}
0 & 0 \\
a_{2,2,1} & a_{2,2,2}
\end{array}\right]
\end{gathered}
$$

where the matrices coefficients are

$$
\begin{aligned}
& d_{1,1,1}=\frac{\alpha-1}{\alpha+1}, d_{2,2,2}=\frac{c_{1} E(\Gamma) \beta-c_{2} G \Sigma}{c_{1} E(\Gamma) \beta+c_{2} G \Sigma}, a_{0,1,1}= \\
& \frac{p k-\zeta}{M \zeta}, a_{0,2,1}=\frac{a_{2} k}{J \zeta}, a_{0,2,2}=-\frac{c_{2} G \Sigma}{c_{1} E(\Gamma) J}, a_{1,1,1}= \\
& -\frac{(\alpha-1)(\zeta+p k)}{(\alpha+1) M \zeta}, a_{2,2,1}=-\frac{p k\left(c_{1} E(\Gamma) \beta-c_{2} G \Sigma\right)}{\zeta\left(c_{1} E(\Gamma) \beta+c_{2} G \Sigma\right) J}, a_{2,2,2}= \\
& -\frac{c_{2} G \Sigma\left(c_{1} E(\Gamma) \beta-c_{2} G \Sigma\right)}{J c_{1} E(\Gamma)\left(c_{1} E(\Gamma) \beta+c_{2} G \Sigma\right)} .
\end{aligned}
$$

Recall that in the above quoted references (concerned by PDE models), the studies was concerned only by the torsional vibrations. Thus the associated NDDE (governing the speed of such vibrations) is scalar which is easier to study compared with (14). And for the physiological model considered in [12], $A_{2}=D_{i}=0$ for $i \in\{1,2\}$ since the model is DDE with one delay.

Setting the numerical values of the physical parameters given in the Appendix and taking $p$ (the parameter deciding for the amplitude of the friction forces $F$ and $\tilde{F}$ ) is left free allows us to the following result.

Proposition 1: $\quad$ - When $\alpha$ is left free and $\alpha=30 p$ then zero is an eigenvalue of algebraic and geometric multiplicity 1 . Moreover, Zero is the only eigenvalue with zero real part and the remaining eigenvalues are with negative real parts. We have a Pitchfork-like bifurcation occurring in ODE, this comes from the Z2 symmetry structure of the system.

- When $\alpha$ takes its physical values $\alpha_{c}$ given in the appendix and $\alpha_{c}=30 p_{c}\left(p_{c}=6.6667\right)$, then zero is an eigenvalue of algebraic multiplicity 2 and of geometric multiplicity 1 . Zero is the only eigenvalue with zero real part and the remaining eigenvalues are with negative real parts. The zero eigenvalue is non-semisimple and the singularity is of Bogdanov-Takens like, see [11].

- The system (14) is formally stable but not asymptotically stable (although there are no characteristic roots with positive real parts).

Analogously to [12] which considers a singular delay system linearly dependent on a parameter, and in the same spirit of the decomposition established in [7] in the goal of computing the normal form for delay systems depending on a parameter, we extend the scheme of computing the center manifold to the case of NDDE depending on parameters and thus look for the system (14) as a perturbation of

$$
\frac{d}{d t} \mathcal{D} x_{t}=\mathcal{L}_{0} x_{t}, \quad \text { where } \quad \mathcal{L}_{0}=\left.\mathcal{L}\right|_{\left\{p=p_{c}, \mu=0\right\}}
$$

Indeed, system (14) can be written as

$$
\begin{aligned}
\frac{d}{d t} \mathcal{D} x_{t}: & =\mathcal{L}_{0} x_{t}+\tilde{\mathcal{F}}_{\mu, p_{\epsilon}}\left(x_{t}\right) \\
& =\mathcal{L}_{0} x_{t}+\left(\mathcal{L}-\mathcal{L}_{0}\right) x_{t}+\mathcal{F}_{\mu, p_{\epsilon}}\left(x_{t}\right)
\end{aligned}
$$

such that

$$
\mathcal{F}_{\mu, p}=\left[\begin{array}{c}
-0.006750 p x_{1}^{3}(t)+0.006682 p x_{1}^{3}(t-2) \\
-1.875 p x_{1}^{3}(t)+1.874998 p x_{1}^{3}(t-1.264911064)
\end{array}\right]
$$

Here we follow the theoretical schemes briefly presented in the Appendix and give computations steps for the equation of the evolution of the problem's solutions on the center variety for system (15).

First, we compute the basis of the generalized eigenspace corresponding to the double eigenvalue $\lambda_{0}=0$.

$$
\Phi(\theta)=\left[\begin{array}{cc}
1-\theta & 1 \\
104351600-104351600 \theta & 104351600
\end{array}\right],
$$

where $\theta \in[-2,0]$. Recall that the adjoint linear equation 
associated to (14) is

$$
\left\{\begin{aligned}
\dot{u}(t)= & D_{1} \dot{u}(t+2)+D_{2} \dot{u}\left(t+\frac{2 \tilde{c}}{c}\right) \\
& -A_{0} u(t)-A_{1} u(t+2)-A_{2} u\left(t+\frac{2 \tilde{c}}{c}\right)
\end{aligned}\right.
$$

with a basis for the generalized eigenspace associated to the double eigenvalue zero is given by

$$
\Psi(\theta)=\left[\begin{array}{cc}
-0.5025082+0.005025011 \xi & 0 \\
1.004179+0.4924583 \xi & 0
\end{array}\right], \xi \in[0,2] .
$$

The associated bilinear form is

$$
\begin{aligned}
(\psi, \varphi)= & \psi(0)\left(\varphi(0)-D_{1} \varphi(-2)-D_{2} \varphi(-1.264911)\right) \\
& +\int_{-2}^{0} \psi(\xi+2) A_{1} \varphi(\xi) d \xi \\
& +\int_{-1.264911}^{0} \psi(\xi+1.264911) A_{2} \varphi(\xi) d \xi \\
& -\int_{-2}^{0} \psi^{\prime}(\xi+2) D_{1} \varphi(\xi) d \xi \\
& -\int_{-1.264911}^{0} \psi^{\prime}(\xi+1.264911) D_{2} \varphi(\xi) d \xi
\end{aligned}
$$

By the introduced bilinear form we can easily check that $(\Psi, \Phi)=I_{d}$, thus the space $C$ can be decomposed as $C=P \bigoplus Q$, where $P=\left\{\varphi=\Phi z ; z \in \mathbb{R}^{2}\right\}$ and $Q=\{\varphi \in C ;(\Psi, \varphi)=0\}$. Recall that each of those subspaces is invariant under the semigroup $T(t)$ and that the matrix $B$ (introduced in the previous section concerned by the theoretical settings) satisfying $\mathcal{A} \Phi=\Phi B$ is given by

$$
B=\left[\begin{array}{cc}
0 & 0 \\
-1 & 0
\end{array}\right]
$$

Let us first set the following decomposition $x_{t}=\Phi y(t)+z(t)$ where $z(t) \in Q$ and $y(t) \in \mathbb{R}^{2}, z(t)=h(y(t))$ and $h$ is some analytic function $h: P \rightarrow Q$. Thus the explicit solution on the center manifold can be obtained by the use of the proven formula in [4], [12] that is

$$
\begin{gathered}
\dot{y}(t)=B y(t)+\Psi(0) \mathcal{F}[\Phi(\theta) y(t)+h(\theta, y(t))] \\
\frac{\partial h}{d y}\{B y+\Psi(0) \mathcal{F}[\Phi(\theta) y+h]\}+\Phi(\theta) \Psi(0) \mathcal{F}[\Phi(\theta) y+h] \\
\quad= \begin{cases}\frac{\partial h}{d \theta}, & -2 \leq \theta \leq 0 \\
\mathcal{L}(h(\theta, y))+\mathcal{F}[\Phi(\theta) y+h(\theta, y)], & \theta=0\end{cases}
\end{gathered}
$$

where $h=h(\theta, y)$ and $\tilde{\mathcal{F}}$ is defined in (16).

Simple computations show that for (15), the evolution of solutions on the center manifold is determined by solving (20) (restricted to $p=p_{c}, \mu=\mu_{c}=0$ ) for $h(\theta, y)$ and then (19) for $y(t)$ (this is done order by order of truncation). It is of important note that $\mathbb{F}$ is an odd function. This fact implies that there is no need to compute $h$. Thus, the third order ODE reduction of the system (13) at $p=p_{c}, \mu=\mu_{c}=0 \Rightarrow \alpha=$ $\alpha_{c}$ is given by

$$
\begin{aligned}
& \dot{y}(t)=\left[\begin{array}{cc}
0 & 0 \\
-1 & 0
\end{array}\right]\left[\begin{array}{l}
y_{1} \\
y_{2}
\end{array}\right] \\
& +\left[\left(\begin{array}{c}
-0.5818668 y_{1}{ }^{3}-0.5366406 y_{1}{ }^{2} y_{2} \\
-0.1336538 y_{1} y_{2}{ }^{2}+0.0002250037 y_{2}{ }^{3} \\
1.162764 y_{1}{ }^{3}+1.072387 y_{1}{ }^{2} y_{2} \\
+0.2670850 y_{1} y_{2}{ }^{2}-0.0004496326 y_{2}{ }^{3}
\end{array}\right)\right.
\end{aligned}
$$

Since our aim is to study the parameter Bifurcations then the computation of the equation of the evolution of the problem's solutions on the center variety for system (16) is required. The same principle applied (i.e. formulas (19) and (20) are used) but the expression of $\mathcal{F}$ change, indeed, the above approximation is made for the system (15) for which $p=p_{c}$, but for the following approximation for (16), $p$ is taken $p=p_{c}+\mu$ where $\mu$ is a small parameter. In the next step, we introduce a small parameter $r$, as a scaling parameter for making a zoom into the neighborhood of the singularity. We introduce the following changes of coordinates $\left\{\mu:=1326.69991 \gamma r^{2}, p=p_{c}+\mu, y_{1}=r^{2} z_{1}, y_{2}=r z_{2}\right\}$ and we scale the time by $t_{\text {old }}=r t_{\text {new }}$ which allows us to the following cubic normal form reduction of (14), (see the Appendix)

$$
\left\{\begin{array}{l}
\dot{z}_{1}=\gamma z_{1}-z_{1} z_{2}^{2} \\
\dot{z}_{2}=-z_{1}
\end{array}\right.
$$

\section{CONCLUding REMARKS}

The main purpose of this paper is a qualitative analysis of a PDE drilling model based on time-delay system approach. In this study we establish the linear stability analysis of the steady-state at the origin for system (14) as well as a qualitative approximation of solutions of the infinite dimensional system (PDE) by solutions of a finite dimensional one (ODE). For system (22), we establish a first integral $I\left(z_{1}, z_{2}\right)=\left(z_{1}+\gamma z_{2}-\frac{1}{3} z_{2}^{3}\right)^{2}$ leading to a Lyapunov function $V\left(z_{1}, z_{2}\right)=z_{1}^{2}+I\left(z_{1}, z_{2}\right)$ asserting that the system is locally asymptotically stable in attraction region $D=\left\{(x, y)\right.$, such that $\left.\gamma<z_{2}^{2}\right\}$. Our future aim will concern the design of appropriate control laws guaranteeing a desired drilling dynamical behavior.

\section{REFERENCES}

[1] M. Ait Babram, O. Arino, and M. L. Hbid. Computational scheme of a center manifold for neutral functional differential equations. $J$. Math. Anal. Appl., 258(2):396-414, 2001.

[2] A. G. Balanov, N.B. Janson, P.V.E. McClintock, and C. H. T. Wang. Bifurcation analysis of a neutral delay differential equation modelling the torsional motion of a driven drill-string. Chaos, Solitons and Fractals, 15:381-394, 2002.

[3] D. A. W. Barton, B. Krauskopf, and R. E. Wilson. Nonlinear dynamics of torsional waves in a drill string model with spacial extent. Journal of Vibration and Control, 16:1049-1065, 2007.

[4] S.A. Campbell. Calculating centre manifolds for delay differential equations using maple. In In Delay Differential Equations: Recent Advances and New Directions. Springer-Verlag, 2009.

[5] J. Carr. Application of Center Manifold Theory. Springer, 1981.

[6] K. Cooke et D. Krumme. Differential-difference equations and nonlinear initial boundary value problems for linear hyperbolic partial differential equations. J. Math. Anal. Appl., 24:372-387, 1968. 
[7] T. Faria and L.T. Magalhães. Normal forms for retarded functionaldifferential equations with parameters and applications to Hopf bifurcation. J. Differential Equations, 122(2):181-200, 1995.

[8] E. Fridman, S. Mondié, and B. Saldivar. Bounds on the response of a drilling pipe model. IMA Journal of Mathematical Control and Information, pages 1-14, 2010.

[9] P. Fu, S-I. Niculescu, and J. Chen. On the Stability of Linear DelayDifferential Algebraic Systems: Exact Conditions via Matrix Pencil Solutions. IEEE Transaction on Automatic Control, 51:1063-1069, 2006.

[10] C. Germay, N. Van De Wouw, H. Nijmeijer, and R. Sepulchre. Nonlinear drilling dynamics analysis. SIAM J. Dynamical Systems, 8(2):527-553, 2005.

[11] J. Guckenheimer and P. Holmes. Nonlinear oscillations, dynamical systems, and bifurcation of vector fields. Springer, 2002.

[12] J. K. Hale and W. Huang. Period doubling in singularly perturbed delay equations. Journal of Differential Equations, 114:1-23, 1994.

[13] J. K. Hale and S. M. Verduyn Lunel. Introduction to functional differential equations, volume 99 of Applied Mathematics Sciences. Springer Verlag, New York, 1993.

[14] Y. Kuznetsov. Elements of applied bifurcation theory; Second edition, volume 112 of Applied Mathematics Sciences. Springer, New York, 1998.

[15] W. Michiels and S-I. Niculescu. Stability and stabilization of timedelay systems, volume 12 of Advances in Design and Control. Society for Industrial and Applied Mathematics (SIAM), Philadelphia, PA, 2007. An eigenvalue-based approach.

[16] H. Mounier. Propriétés structurelles des systèmes linéaires a retard : Aspects théorique et pratique. Thèse de l'Université Paris-Sud, (3888):148, 1995.

[17] E. M. Navarro-López and D. Cortés. Sliding-mode control of a multidof oilwell drillstring with stick-slip oscillations. Proceeding of the 2007 American Control Conference, pages 3837 - 3842, 2007.

[18] E. M. Navarro-López and R. Suárez. Modelling and analysis of stickslip behavior in a drillstring under dry friction. Congreso anual de la AMCA 2004, pages 330 - 335, 2004.

[19] E. M. Navarro-López and R. Suárez. Practical approach to modelling and controlling stick-slip oscillations in oilwell drillstring. Proceeding of the 2004 IEEE, International Conference on Control Applications, 10(5):3162 - 3174, 2004.

[20] Eva M. Navarro-López. An alternative characterization of bit-sticking phenomena in a multi-degree-of-freedom controlled drillstring. Nonlinear Analysis: Real World Applications, 10(5):3162 - 3174, 2009.

[21] S-I. Niculescu. Delay Effects on Stability, A Robust Control Approach. Springer, Lecture Notes in Control And Information Sciences, 2001.

[22] Tucker R. W. and Wang C. On the effective control of torsional vibrations in drilling systems. Journal of Sound and Vibration, 224(1):101-122, 1999.

[23] V. Rasvan and S-I. Niculescu. Oscillations in lossless propagation models: a liapunov-krasovskii approach. IMA J. of Math. Contr. and Info., 19:157-172, 2002.

[24] Th. Richard, Ch. Germay, and E. Detournay. A simplified model to explore the root cause of stick-slip vibrations in drilling system with drag bits. Appl. Math. Comput., 305:432-456, 2007.

[25] P. Rouchon. Flatness and stick-slip stabilization. Technical Report, 492:1-9, 1998.

[26] B. Saldivar, S. Mondié, J-J. Loiseau, and V. Rasvan. Stick-slip oscillations in oillwell drilstrings: Distributed parameter and neutral type retarded model approaches. IFAC 18th World Congress Milano (Italy) August 28 - September 2, 2011, pages 283-289, 2011.

[27] M. Weedermann. Normal forms for neutral functional differential equations. In Topics in functional differential and difference equations (Lisbon, 1999), volume 29 of Fields Inst. Commun., pages 361-368. Amer. Math. Soc., Providence, RI, 2001.

[28] M. Weedermann. Hopf Bifurcation calculations for scalar delay differential equations. Nonlinearity, 19:2091-2102, 2006.

\section{APPENDIX}

\section{A. Graphical Illustration}

The projection of the dynamics on the center manifold for the critical value $p=p_{c}$ is given in Figure 1 and $p=p_{c}+\mu$ is given in Figure 2 and 3. Figure 4 gives the state $z_{1}$ response for various values of $\gamma$.

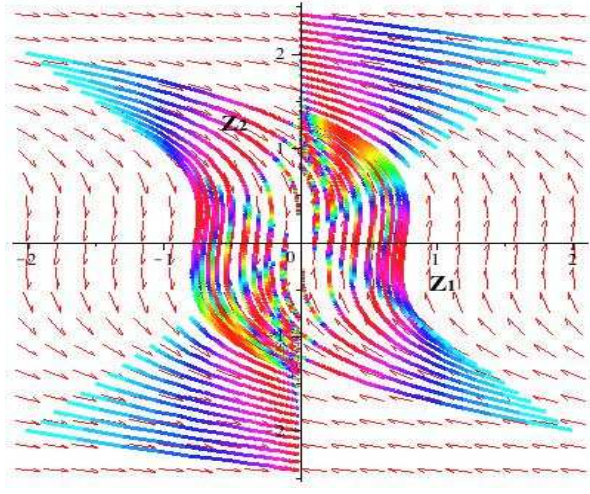

Fig. 1. Phase portrait of the system (22) $\left.\right|_{\gamma=0}$ that is $p=p_{c}$

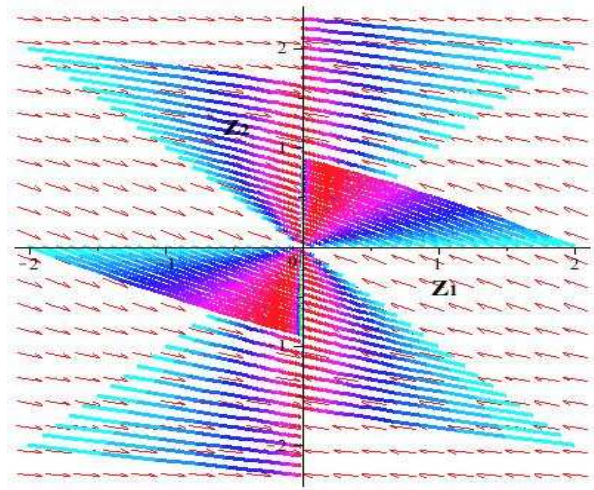

Fig. 2. Phase portrait of the system (22) $\left.\right|_{\gamma=-2}$ that is $p=p_{c}+\mu$

\section{B. Numerical Settings}

\begin{tabular}{|c|c||c|c|}
\hline Parameter & Value & Parameter & Value \\
\hline $\mathrm{G}$ & $80 \mathrm{GPa}$ & $\mathrm{E}$ & $200 \mathrm{GPa}$ \\
\hline$\rho$ & $8000 \mathrm{Kg} / \mathrm{m}^{3}$ & $\mathrm{r}$ & $6 \mathrm{~cm}$ \\
\hline$\Gamma$ & $35 \mathrm{~cm}^{2}$ & $\Sigma$ & $19 \mathrm{~cm}^{4}$ \\
\hline $\mathrm{L}$ & $3000 \mathrm{~m}$ & $\mathrm{M}$ & $40000 \mathrm{Kg}$ \\
\hline$\alpha$ & $200,025 \mathrm{~kg} / \mathrm{s}$ & $\beta$ & $2000 \mathrm{Nms}$ \\
\hline$k$ & 0,3 & $\zeta$ & 0,01 \\
\hline
\end{tabular}

\section{Proof of Proposition 1}

We compute the characteristic equation associated with (14) which gives

$$
\begin{aligned}
& \operatorname{det}(\Delta)=F_{1}(\lambda) F_{2}(\lambda, p) \\
& =\left[\left(0.9999990 \mathrm{e}^{-1.264911064 \lambda}-1\right) \lambda\right. \\
& \left.-0.0000066 \mathrm{e}^{-1.264911064 \lambda}-0.0000066\right] \times \\
& {\left[\left(-1+0.990049 \mathrm{e}^{-2 \lambda}\right) \lambda\right.} \\
& \left.+(-0.000024-0.0007 p) \mathrm{e}^{-2 \lambda}+0.00075 p-0.000025\right]
\end{aligned}
$$

Considering separately these factors simplifies the analysis. Let us consider the first factor

$$
\begin{aligned}
F_{1}(\lambda) & =\left(1-0.9999990 \mathrm{e}^{-1.264911064 \lambda}\right) \lambda \\
& +0.0000066+0.0000066 \mathrm{e}^{-1.264911064 \lambda}
\end{aligned}
$$

it is a scalar first order quasi-polynomial, by substituting $\lambda=I \omega$ we show that there are no spectral values with zero 


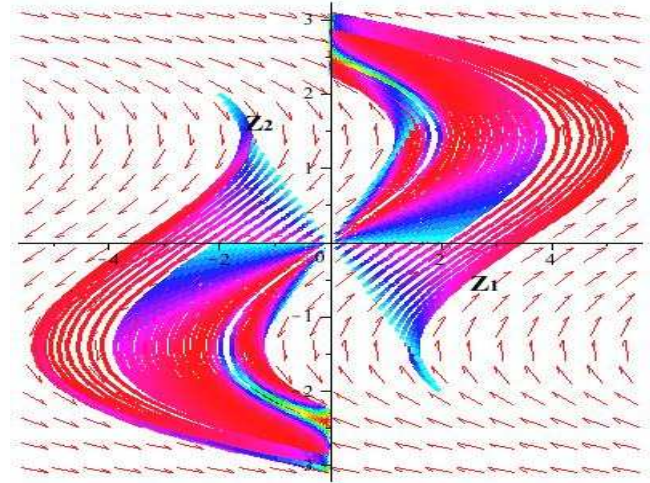

Fig. 3. Phase portrait of the system (22) $\left.\right|_{\gamma=2}$ that is $p=p_{c}+\mu$

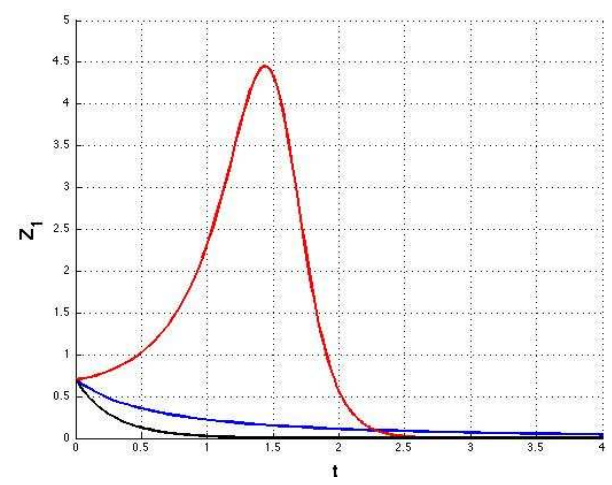

Fig. 4. The State response $z_{1}$ for (22) (red) $\gamma=2$, (black) $\gamma=0$ and (blue) $\gamma=-2$

real part. This scalar quasi-polynomial satisfy the conditions for a Pontryagin theorem for scalar quasi-polynomial which leads to prove that all spectral values have negative real part, see [28]. Let us now consider the second factor

$$
\begin{aligned}
F_{2}(\lambda, p) & =\left(0.990049 \mathrm{e}^{-2 \lambda}-1\right) \lambda+0.00075 p-0.000025 \\
& +(-0.000024-0.000742 p) \mathrm{e}^{-2.0 \lambda}
\end{aligned}
$$

By the same way $(\lambda=I \omega)$ we show that, apart from $\omega_{0}=0$, there are no spectral values with zero real part. Moreover, $p=p_{c}=6.666749999$ is the only possible value for $p$ leading to a spectral value in zero. Let us adopt the following parametrization of the problem $\mu=\alpha-30 p$.

$$
\begin{aligned}
F_{2}(\lambda, \mu, p)= & {\left[(40000 \mu+1200000 p-40000) \mathrm{e}^{-2 \lambda}\right.} \\
& -1200000 p-40000 \mu-40000] \lambda \\
& +\left(1-\mu-30 p \mu-900 p^{2}\right) \mathrm{e}^{-2 \lambda} \\
& -1-\mu+30 p \mu+900 p^{2} .
\end{aligned}
$$

Computations show that $\mu=\mu_{c}=0$ leads to a first spectral value on the imaginary axis $\lambda_{1}=0$, thus we have a like Pitchfork bifurcation occurring in ODE. Indeed, this comes from the $\mathbb{Z}_{2}$ symmetry structure of the system. When additionally $p=p_{c}=6.666749999\left(\mu=\mu_{c}=\right.$ $\left.0 \Rightarrow \alpha=\alpha_{c}=30 \times 6.666749999\right)$ then $\lambda_{2}=0$, thus we are in case of a double root at zero of non-semisimple type since $\mathcal{N}\left(\lambda_{0} I_{d}-A\right)$ have only one eigenvector, namely $v_{0}=\left[\begin{array}{c}1 \\ 103851200\end{array}\right]$. Moreover, in that case and for the same reasons as for the first factor, the remaining roots of $F_{2}$ have negative real parts and thus, the spectra of $\mathcal{A}$ contains two chains situated in the left half plane (first chain located near $\operatorname{Re}(\lambda)=-0.00005$ and the second chain is located near $\operatorname{Re}(\lambda)=-0.0000007$ ) and an isolated (double) spectral value in zero.

In conclusion, the system is formally stable but not asymptotically stable (although there are no characteristic roots with positive real parts) and the singularity is of BogdanovTakens like, see [14], [11].

\section{Model reduction}

Generally speaking, Functional differential equations (FDE) share some properties with ordinary differential equations (ODE). By this section we would like to present how this can be done in approximating the behavior of the dynamics of a neutral delay differential equation (NDDE) by the one of a simpler ODE. Indeed, having computed the projection of the NDDE to a center manifold which leads to an ODE, the normal forms theory comes to simplify its structure without any loss in the qualitative dynamic in a neighborhood of the state equilibrium.

Let us consider the general discretely delayed autonomous first order nonlinear system of Neutral type where we separate its linear and nonlinear quantities as follow

$$
\begin{aligned}
& \frac{d}{d t}\left[x(t)+\sum_{k=1}^{n} A_{k} x\left(t-\tau_{k}\right)\right]= \\
& \sum_{k=0}^{n} B_{k} x\left(t-\tau_{k}\right)+\mathcal{F}\left(x(t), \ldots, x\left(t-\tau_{n}\right)\right)
\end{aligned}
$$

where $A_{i}, B_{j}$ are $n \times n$ real valued matrix and the delays $\tau_{k}$ are ordered such that $\tau_{j}<\tau_{j}$ when $i<j$ and let $\tau_{n}=r$.

The latter system can be written

$$
\frac{d}{d t} \mathcal{D} x_{t}=\mathcal{L} x_{t}+\mathcal{F}\left(x_{t}\right)
$$

where $x_{t} \in C=C\left([-r, 0], \mathbb{R}^{n}\right), x_{t}(\theta)=x(t+\theta), \mathcal{D}, \mathcal{L}$ are bounded linear operators such that $\mathcal{L} \phi=\sum_{k=0}^{n} B_{k} \phi\left(-\tau_{k}\right)$, $\mathcal{D} \phi=\phi(0)+\sum_{k=1}^{n} A_{k} \phi\left(-\tau_{k}\right)$ and $\mathcal{F}$ is sufficiently smooth function mapping $C$ into $\mathbb{R}^{n}$ with $\mathcal{F}(0)=D \mathcal{F}(0)=0$. We point out that the linear operators $\mathcal{D}$ and $\mathcal{L}$ can be written in the integral form by $\mathcal{L} \phi=\int_{-r}^{0} d \eta(\theta) \phi(\theta)$ and $\mathcal{D} \phi=$ $\phi(0)+\int_{-r}^{0} d \mu(\theta) \phi(\theta)$, where $\mu$ and $\eta$ are two real valued $n \times n$ matrix.

The linearized equation of (24) is given by

$$
\frac{d}{d t} \mathcal{D} x_{t}=\mathcal{L} x_{t}
$$

for which the operator solution $\mathcal{T}(t)$ defined by $\mathcal{T}(t)(\phi)=$ $x_{t}(., \phi)$ such that $x_{t}(., \phi)(\theta)=x(t+\theta, \phi)$ for $\theta \in[-r, 0]$ is a strongly continuous semigroup with the infinitesimal generator given by $\mathcal{A}=\frac{d \phi}{d \theta}$ with the domain 


$$
\operatorname{Dom}(\mathcal{A})=\left\{\phi \in C: \frac{d \phi}{d \theta} \in C, \mathcal{D} \frac{d \phi}{d \theta}=\mathcal{L} \phi\right\}
$$

It is also known that $\sigma(\mathcal{A})=\sigma_{p}(\mathcal{A})$ and the spectrum of $\mathcal{A}$ consists of complex values $\lambda \in \mathbb{C}$ which satisfy the characteristic equation $p(\lambda)=\operatorname{det} \Delta(\lambda)=0$, see [15] for further details.

Let us denote by $\mathcal{M}_{\lambda}$ the eigenspace associated with $\lambda \in$ $\sigma(\mathcal{A})$. We define $C^{*}=C\left([-r, 0], \mathbb{R}^{n *}\right)$ where $\mathbb{R}^{n *}$ is the space of n-dimensional row vectors and consider the bilinear form on $C^{*} \times C$ which is proposed in [13]

$$
\begin{aligned}
(\psi, \phi) & =\phi(0) \psi(0)-\int_{-r}^{0} d\left[\int_{0}^{\theta} \psi(\tau-\theta) d \mu(\tau)\right] \\
& +\int_{-r}^{0} \int_{0}^{\theta} \psi(\tau-\theta) d \eta(\theta) \phi(\tau) d \tau
\end{aligned}
$$

and let $\mathcal{A}^{T}$ be the transposed operator of $\mathcal{A}$, i.e., $(\psi, \mathcal{A} \phi)=$ $\left(\mathcal{A}^{T} \psi, \phi\right)$. The following Theorem [13] permits the decomposition of the space $C$.

Theorem 2: Let $\Lambda$ be a nonempty finite set of eigenvalues of $\mathcal{A}$ and let $P=\operatorname{span}\left\{\mathcal{M}_{\lambda}(\mathcal{A}), \lambda \in \Lambda\right\}$ and $P^{T}=\operatorname{span}\left\{\mathcal{M}_{\lambda}\left(\mathcal{A}^{T}\right), \lambda \in \Lambda\right\}$. Then $P$ is invariant under $\mathcal{T}(t), t \geq 0$ and there exists a space $\mathcal{Q}$, also invariant under $\mathcal{T}(t)$ such that $C=P \bigoplus Q$. Furthermore, if $\Phi=$ $\left(\phi_{1}, \ldots, \phi_{m}\right)$ forms a basis of $P, \Psi=\operatorname{col}\left(\psi_{1}, \ldots, \psi_{m}\right)$ is a basis of $P^{T}$ in $C^{*}$ such that $(\Phi, \Psi)=I d$, then

$$
\begin{aligned}
& Q=\{\phi \in C \backslash(\Psi, \phi)=0\} \text { and } \\
& P=\left\{\phi \in C \backslash \exists b \in \mathbb{R}^{m}: \phi=\Phi b\right\} .
\end{aligned}
$$

Also, $\mathcal{T}(t) \Phi=\Phi e^{B t}$, where $B$ is an $m \times m$ matrix such that $\sigma(B)=\Lambda$.

Let us consider the extension of the space $C$ that contains continuous functions on $[-r, 0)$ with possible jump discontinuity at 0 , we denote this space $B C$. A given function $\xi \in B C$ can be written $\xi=\varphi+X_{0} \alpha$, where $\varphi \in C$, $\alpha \in \mathbb{R}^{n}$ and $X_{0}$ is defined by $X_{0}(\theta)=0$ for $-r \leq \theta<0$ and $X_{0}(0)=I d_{n \times n}$. Then Hale-Verduyn Lunel bilinear form [13] can be extended to the space $C^{*} \times B C$ by $\left(\psi, X_{0}\right)=\psi(0)$ and the infinitesimal generator $\mathcal{A}$ extends to an operator $\tilde{\mathcal{A}}$ (defined in $C^{1}$ ) onto the space $B C$ as follow

$$
\tilde{\mathcal{A}} \phi=\mathcal{A} \phi+X_{0}\left[\mathcal{L} \phi-\mathcal{D} \phi^{\prime}\right]
$$

Under the above consideration one can write equation (24) as an abstract ODE

$$
\dot{x}_{t}=\tilde{\mathcal{A}} x_{t}+X_{0} \mathcal{F}\left(x_{t}\right),
$$

see [27], [7]. Thanks to the projection $\Pi: B C \rightarrow P$ such that $\Pi\left(\varphi+X_{0} \alpha\right)=\Phi[(\Psi, \varphi)+\Psi(0) \alpha]$ hence $x_{t}=\Phi y(t)+z_{t}$ where $y(t) \in \mathbb{R}^{m}$ and then equation (24) can be split to

$$
\begin{aligned}
& \dot{y}=B y+\Psi(0) F(\Phi y+z) \\
& \dot{z}=\tilde{\mathcal{A}}_{Q}+(I-\Pi) X_{0} \mathcal{F}(\Phi y+z),
\end{aligned}
$$

and our interest will be focused only on the first equation after writing $z$ as a function of $y$. The way to do that is the center manifold technique.

\section{E. Center Manifold}

Definition 3: Given a $C^{1}$ map $h$ from $\mathbb{R}^{2}$ into $Q$. The graph of $\mathrm{h}$ is said to be a local manifold associated to (24) if $h(0)=D h(0)=0$ and there exists a neighborhood $V$ of $0 \in \mathbb{R}^{2}$ such that for each $\xi \in V$, there exists $\delta=\delta(\xi)>0$ and the solution $x$ of (24) with initial data $\Phi \xi+h(\xi)$ exists on the interval $]-\delta-r, \delta\left[\right.$ and it is given by $x_{t}=\Phi y(t)+h(y(t))$ for $t \in[0, \delta[$ where $y(t)$ is the unique solution of the ODE

$$
\begin{aligned}
& \dot{y}=B y+\Psi(0) F(\Phi y+h(y)) \\
& y(0)=\xi
\end{aligned}
$$

For some particular cases of the matrix $B$ analytic characterization of the center manifold was the subject of few studies, for instance in the case of Bogdanov-Takens singularity one can find the characterization of the function $h$ in [1]. Note also that in the above definition it assumed that $y \in \mathbb{R}^{2}$, but it stills correct for $y \in \mathbb{R}^{m}$ for instance we quote the case of a Double Hopf Bifurcation, the most important is to make a decomposition which separate between the eigenspace of all imaginary eigenvalues (real part equal to zero) and the space of the remaining spectral values which are assumed to have negative real parts.

Note that in our study we chose to consider and make the necessary adaptations to the method and the scheme of center manifold computations distinctly presented by Campbell in [4] and originally made for delay differential equations.

In what follow we give the outlines of the normal forms approach taking into account that $y \in \mathbb{R}^{2}$ this simple case is of considerable importance since this is typically the case of Hopf bifurcation or Bogdanov-Takens one.

\section{F. Normal Forms Theory}

It is well known in the field of qualitative theory of ODE that normal forms approach is a powerful tool in the study of the local dynamics in neighborhood of singular points. Among other problems local bifurcation and stability analysis benefit from this theory.

Let $x=\left(x_{1}, x_{2}\right) \in \mathbb{R}^{2}$ and $f\left(x_{1}, x_{2}\right) \in \mathbb{R}\left[x_{1}, x_{2}\right] \times$ $\mathbb{R}\left[x_{1}, x_{2}\right]$ and consider the general planar system

$$
\dot{x}=L x+f(x)=L x+f_{2}(x)+f_{3}(x)+\ldots,
$$

where $L x$ represents the linear part, $L$ the Jacobian matrix associated to system (31) and $f_{k}(x)$ denotes the $k^{\text {th }}$ order vector homogeneous polynomials of $x$. We assume that the system admits an equilibrium at the origin $O$. The essential idea of the Normal Form theory is to find a near identity transformation

$$
x=y+h(y)=y+h_{2}(y)+h_{3}(y)+\ldots,
$$

by which the resulting system

$$
\dot{y}=L y+g(x)=L y+g_{2}(y)+g_{3}(y)+\ldots,
$$

becomes as simple as possible. In this sense, the terms that are not essential in the local dynamical behavior are removed from the analytical expression of the vector field. Let us denote by $h_{k}(y)$ and $g_{k}(y)$ the $k^{t h}$ order vectors 
homogeneous polynomials of $y$. According to Takens normal form theory, we define an operator as follows:

$$
L_{k}: H_{k} \rightarrow H_{k}, \quad U_{k} \in H_{k} \mapsto L_{k}\left(U_{k}\right)=\left[U_{k}, u_{1}\right] \in H_{k}
$$

where $u_{1}=L y$ is the linear part of the vector field and $H_{k}$ denotes a linear vector space containing the $k^{t h}$ degree homogeneous vector polynomials of $y=\left(y_{1}, y_{2}\right)$. The operator $[.,$.$] is called the Lie Bracket, defined by$

$$
\left[U_{k}, u_{1}\right]=L U_{k}-D\left(U_{k}\right) u_{1}
$$

where $D$ denotes the frechet derivative.

Next, we define the spaces $R_{k}$ and $K_{k}$ as the range of $H_{k}$ and the complementary space of $R_{k}$ respectively. Thus, $H_{k}=R_{k}+K_{k}$ and one can then choose bases for $K_{k}$ and $R_{k}$. The normal form theorem determines how it is possible to reduce the analytic expression of the vector field (see Gukenheimer and Holmes book [11]). The authors give explicitly an analysis for the quadratic and the cubic cases. Consequently, a vector homogeneous polynomial $f_{k} \in H_{k}$ can be split into two parts, such that one of them can be spanned in $K_{k}$ and the remaining part in $R_{k}$.

Normal form theory shows that the part belonging to $R_{k}$ can be eliminated and the remaining part can be retained in the normal form. By the equations (31), (32) and (33), we can obtain algebraic equations one order after another. 